\title{
A Power Control Mechanism Based on Priority Grouping for Small Cell Networks
}

\author{
Antonios Alexiou \\ Computer Engineering and Informatics Department \\ University of Patras \\ Patras, Greece \\ alexiua@ceid.upatras.gr
}

\author{
Dimitrios Billios, Christos Bouras \\ Computer Technology Institute \& Press "Diophantus" \\ Computer Engineering and Informatics Department \\ University of Patras \\ Patras, Greece \\ bilios@ceid.upatras.gr,bouras@cti.gr
}

\begin{abstract}
Future mobile networks following Long Term Evolution (LTE) will be characterized by demanding services, in terms of bandwidth and delay, already dominant in wired networks, such as cloud-computing, High Definition of anything (HDOA) and highly-interactive online games. Small Cell technology is one of the key-technologies aiming to address the need for ultra-high performance in future mobile networks. This paper is focused on efficient power control in Small Cell Networks (SCNs). In particular, we propose a power control mechanism for efficient power allocation in SCNs. The proposed mechanism efficiently controls systems' interference while on the other hand guarantees home user QoS. We introduce the methodology of Priority Grouping, in which home users in the topology is assigned to one of the available groups with different priorities in terms of power requirements and requested traffic load. The mechanism dynamically updates the Home evolved Node B power setting based on the topology of the macro and home users in real time. Additionally, depending on the examined traffic scenario the mechanism can provide better protection (in terms of interference) either on macro users or on home users.
\end{abstract}

Keywords- small cells; power control; radio resource allocation; radio resource management; LTE; femto cell

\section{INTRODUCTION}

The emergence of new data and video services coupled with an increase in the number of user equipments such as smart-phones and tablets, has forced mobile operators to examine new ways for increasing coverage, boosting data rates and lowering capital and operating expenditures (CAPEX and OPEX) of their mobile networks [1]. A promising way to deal with these demands is the concept of Small Cell Networks (SCN). Small Cells have a strong potential for increasing the efficiency, cell coverage and network capacity of next-generation mobile networks while on the other hand the deployment cost for the service provider is kept in extremely low level.

Although SCNs provide several benefits for operators and users alike, their massive deployment comes with a number of technical challenges. Notably, an important and detrimental problem facing $\mathrm{SCNs}$ is the presence of interference among neighboring SNCs, and between the SNCs and the macrocell LTE network [2]. Several solutions are presented in the bibliography about how to mitigate interference in co-channel femto-to-macro Downlink (DL) interference [3][4][5][6][7]. These studies have consistently shown that a coverage-hole exists when co-channel femto cells are deployed in a macrocell overlay network. Additionally, the studies have shown that although a femto cell can cause interference to other users in the system, the interference can be well controlled on both the downlink and uplink if proper interference management techniques are used [8][9].

Another way to deal with interference in SCNs is through Power Control Optimization. The main purpose of power control is to minimize the transmitted power, thus avoiding unnecessary high power levels and eliminating interference. By appropriately adjusting the downlink transmission power per Resource Blocks (RB) that is required to obtain a target bit rate in femto cells, the overall generated interference in the SCN could be significantly reduced. In other words, Home evolved NodeB (HeNB) adjusts its transmission power so as to satisfy home user (HUE) Quality of Service (QoS) while protecting macrocell users (MUEs) in its vicinity by keeping the interference below a threshold.

A major portion of the existing literature has investigated the interference management issues of integrated LTE and Small Cell deployments [3][4][5][6][7][8][9]. However, the area of Power Control in Small Cell networks is not thoroughly investigated in the literature. A joint admission and power control algorithm where the small cells can determine jointly their admissibility and transmit powers autonomously is introduced in [11]. Authors in [12] present a study of Power control in two-tier femto cell networks where an algorithm that reduces transmission powers of the strongest femto cell interferers is proposed. A distributed algorithm for downlink resource allocation in multicarrier small cell networks is also introduced in [13]. In this algorithm, each home base station selects the resource allocation strategy to maximize a surplus function comprising both, own cell utility and interference prices (that reflect the interference that is caused to neighboring cells).

Although the above presented approaches provide an adequate solution to the problem of power control and interference management in SCNs, they present a number of drawbacks that make difficult their widespread adoption in SCNs, since they don't cover all the possible topology and traffic scenarios that can be realized in SCNs. In this paper, we propose a power control mechanism for efficient power allocation in SCNs. The proposed mechanism efficiently 
controls systems' interference while on the other hand guarantees user QoS. The mechanism dynamically updates the HeNB power setting in real time based on the topology of the macro and home users as well as the requested traffic scenario by the users. To achieve this, we introduce the methodology of Priority Grouping, in which each HUE in the topology is assigned to one of the available groups with different priorities in terms of power requirements and requested traffic load. The use of Priority Groups can result to a combined usage of more than one power control algorithms from the HeNB depending on the traffic scenario. Furthermore, depending on the examined traffic scenario the mechanism can provide better protection (in terms of interference) either on macro users or on home users. The proposed mechanism is evaluated through a user-friendly graphical tool designed to reproduce and calculate the optimal transmission parameters, via a graphical representation of the entire topology for a highly customizable network configuration.

The paper is structured as follows: Section II provides a brief description of Power Control in SCNs. Section III presents the proposed power control mechanism for SCNs, while Section IV is dedicated to the evaluation of the proposed. Finally, the planned next steps as well as the concluding remarks are briefly described in Section V.

\section{POWER CONTROL IN LONG TERM EVOLUTION SMALL Cell Home Base Stations}

In this section, we focus on the description of the power control algorithms performed on a Frequency Division Duplex LTE Small Cell HeNB. A typical topology of such a network is presented in Fig. 1. In the LTE terminology, Home eNBs (HeNBs) are also known as femto cells, thus the terms HeNB and femto cell will be used interchangeably in this paper.

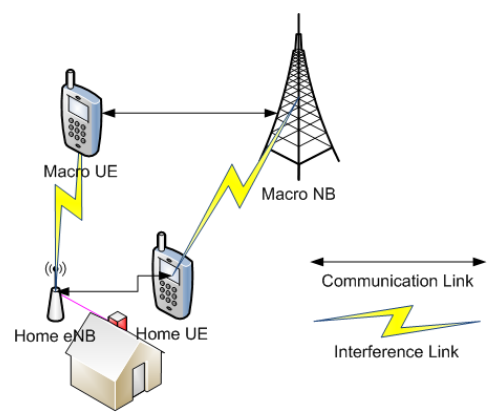

Figure 1. Topology of an FDD LTE Small Cell HeNB

The most common algorithms for HeNB Downlink Power Control as defined by the 3GPP are presented below:

- Algorithm 1: Fixed HeNB power setting [9]

- Algorithm 2: Smart power control based on interference measurement from macro NodeB [9], [14]

- Algorithm 3: HeNB power control based on HeNBMUE path loss [9]
In Algorithm 1, Network Operator typically sets HeNB transmission power manually. HeNB power remain stable and does not depend on the changes appeared in the topology and the traffic scenario.

In Algorithm 2, HeNB adjusts its maximum DL transmit power as a function of air interface measurements to avoid interfering with macro cell UEs. The HeNB adjusts its maximum transmit power according to the following formula:

$$
P_{t x}=\max \left(\min \left(\alpha \times\left(C R S \hat{E}_{C}+10 \log \left(N_{R B}^{D L} \times N_{S C}^{R B}\right)\right)+\beta, P_{\max }\right), P_{\min }\right)
$$

where:

parameters $P_{\max }$ and $P_{\min }$ is the maximum and minimum HeNB transmit power settings, $C R S \hat{E}_{c}$ is measured in $\mathrm{dBm}$, which is the RSRP per resource element present at the Home $\mathrm{BS}$ antenna connector received from the strongest co-channel macro cell. $N_{R B}^{D L}$ is the number of downlink resource blocks in the HeNB channel. $N_{s c}^{R B}$ is the number of subcarriers in a resource block $\left(N_{s c}^{R B}=12\right)$. Parameter $\alpha$ is a linear scalar that allows altering the slope of power control mapping curve, $\beta$ is a parameter expressed in $\mathrm{dB}$ that can be used for altering the exact range of $C R S \hat{E}_{c}$ covered by dynamic range of power control. Parameters $P_{\text {min }}, \alpha$, and $\beta$ are considered to be HeNB configuration parameters, and $P_{\max }$ corresponds to the HeNBs maximum transmit power capability.

According to Algorithm 3, HeNB adjusts the downlink transmit power by taking into account the path loss between the HeNB and an outdoor neighbor MUE including penetration loss in order to provide better interference mitigation for the MUE while maintaining sufficient HeNB coverage for HUEs. HeNB sets the transmit power of reference signal $P_{t x}$ as follows:

$$
P_{t x}=\operatorname{MEDIAN}\left(P_{m}+P_{o f f s e t}, P_{t x_{-} u p p}, P_{t x_{-} l o w}\right)[d B m]
$$

where:

$P_{m}(\mathrm{dBm})$ is RSRP from the nearest Macro evolved NodeB (MeNB) measured by the HeNB. $P_{m}$ is dependent on path loss which includes the penetration loss between the nearest MeNB and the HeNB. $P_{\text {offset }}(\mathrm{dB})$ is the power offset described in equation 3 in detail and $P_{t x_{-} u p p} / P_{t x_{-} \text {low }}(\mathrm{dBm})$ is the upper/lower limit value for the transmit power of the reference signal. The maximum and the minimum total transmit power of HeNB should follow HNB in [10].

The HeNB can also set the maximum downlink transmit power in proportion to the transmit power of the reference signal. As the RSRP decreases, which means the HeNB is located close to the edge of the macro cell, the transmit power should be small in order to mitigate the downlink interference to the MUE.

$P_{\text {offset }}$ above should be defined based on path loss between the HeNB and the MUE. The path loss may consist of indoor path loss between the HeNB and cell edge of HeNB cell and the penetration loss. Therefore, $P_{\text {offset }}$ should be formulated as in (3): 


$$
P_{\text {offset }}=\operatorname{MEDIAN}\left(P_{\text {offset_o }_{-}}+K^{*} L E, P_{\text {offset__max }}, P_{\text {offset_min }}\right)
$$

where:

$P_{\text {offset o }}(\mathrm{dB})$ is a predetermined power offset value corresponding to the indoor path loss. Typical value range between 50 and $100 \mathrm{~dB}$, and can be determined by the averaged measurement value. $K$ is an adjustable positive factor can be determined by the priority of HeNB operation. This value should be high to increase the total transmit power (MeNB is more acceptable to higher interference) and low to reduce the interference to MeNB operation. $L E$ (dB) is estimated penetration loss as below. $P_{\text {offset max }} / P_{\text {offset min }}$ (dB) is the maximum/minimum value of the $P_{\text {offset }}$ by which the estimated and calculated $P_{\text {offset }}$ can be prevented from being too large or too small. This value is dependent of the actual wall penetration loss plus $P_{\text {offset_o. The typical wall }}$ penetration loss ranges between 10 and $30 \mathrm{~dB}$.

A comparative presentation of the above algorithms is presented in Table I.

TABLE I. COMPARISON OF ALGORITHMS

\begin{tabular}{|c|c|c|c|}
\hline Criteria & Algorithm 1 & Algorithm 2 & $\begin{array}{c}\text { Algorithm } \\
3\end{array}$ \\
\hline $\begin{array}{l}\text { Better protection to } \\
\text { MUES }\end{array}$ & No & Yes & Yes \\
\hline Signalling overhead & Low & Low & High \\
\hline $\begin{array}{l}\text { Can exceed max Transmit } \\
\text { power }\end{array}$ & No & No & Yes \\
\hline $\begin{array}{l}\text { Better performance for } \\
\text { high traffic demand users }\end{array}$ & No & Yes & Yes \\
\hline $\begin{array}{l}\text { Can adjust to network } \\
\text { conditions (macro power, } \\
\text { number of UE) }\end{array}$ & No & Yes & Yes \\
\hline Complexity & Low & Medium & High \\
\hline
\end{tabular}

\section{Proposed POWER CONTROL MECHANISM}

In this section, we present a power control mechanism that makes efficient use of the above presented algorithms so as to provide an efficient solution to the problem of power control in future SCNs. A block diagram of the mechanism is presented in Fig. 2 and the description of the mechanism in pseudo code is presented in Fig. 3. Our mechanism senses the topology and traffic scenario requirements in real time and selects each time the algorithm(s) that best fit to the current topology instance and traffic scenario. We introduce the methodology of Priority Grouping, in which each HUE in the topology is assigned to one of the available groups with different priorities in terms of power requirements and traffic load. The use of Priority Groups can result to a combined usage of more than one power control algorithms from the HeNB depending on the traffic scenario. This in turn means that HeNB performs a more efficient power allocation and interference management.

More specifically, the scheme consists of four distinct operation phases. These are: Initialization phase, Algorithm Selection phase, Power Computation phase, and Event Scheduling phase. HeNB is the responsible node of the SCN architecture for the operation of this mechanism.
Regarding the Initialization phase (Fig. 2), at first, the mechanism categorizes the HUEs that reside in the topology based on their traffic requirements to a predefined number of $N_{G}$ priority groups. In each of those groups, a target mean HUE throughput $\left(T h_{\text {target }}\right)$ is set. For example, a HUE with heavy traffic requirements (ex real time streaming) is categorized to a priority group with high value of $T h_{\text {target }}$, while a HUE with reduced traffic requirements (ex. http browsing) is categorized to a priority group with low value of $T h_{\text {target }}$. What we want to achieve with this categorization is to distinguish between the HUEs that their traffic scenario require increased power resources and those HUEs that have reduced power requirements. In addition, we assume that the maximum available power $\left(P_{\text {available }}\right)$ is known to the HeNB.

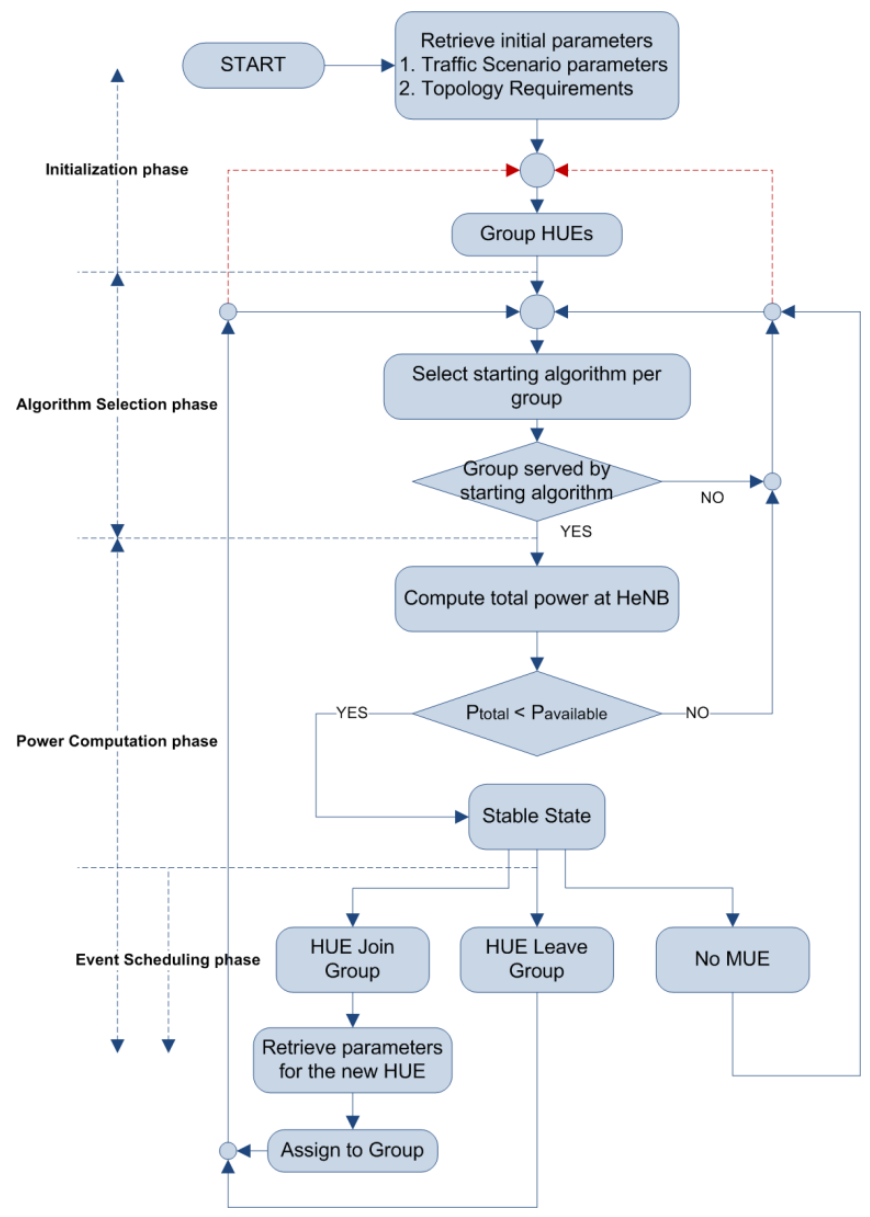

Figure 2. Block diagram of the proposed mechanism

In Algorithm Selection phase, the power control algorithm that will firstly be adopted by each of the $N_{G}$ groups is selected. At this stage, the mechanism selects the algorithm that requires minimum initial power as the starting algorithms of the group. Following this, in each group the mechanism checks if the starting algorithm could serve the dedicated for the group traffic load, taking into account the mean HUE throughput per group $\left(T h_{\text {target }}\right)$, the number of HUEs in each group as well as their location within the femto cell. If the selected algorithm in each group has 
enough power to support the traffic requirements of the group the mechanism enters the Power Computation phase. Alternatively, the mechanism selects another starting algorithm(s) for the group(s) that their traffic load cannot be served by the previously selected starting algorithm(s).

In Power Computation phase, the total power $\left(P_{\text {total }}\right)$ is calculated in HeNB as a sum of the required power in each of the $N_{G}$ priority groups. Following this, $P_{\text {total }}$ is compared to $P_{\text {available }}$ in order to secure that the system's total power is kept in an acceptable level. If $P_{\text {total }}<P_{\text {available }}$ the mechanism enters the stable state which means that all users in the topology are served well with respect to their traffic requirements. In case where $P_{\text {total }}>P_{\text {available }}$, the mechanism need to reduce the system's total power either:

- by proceeding to the reselection of the power control algorithm per group or

- by performing a total system regrouping (reduce number of groups or re-characterise HUE keeping the NG as is).

At this point it should be noted that the latter procedure should be avoided since it greatly increases mechanism's complexity. However, in cases where system's total power is extremely high, group reorganization is necessary as this means that the selected priority grouping is not accurately corresponding to the traffic requirements and system's capacity.

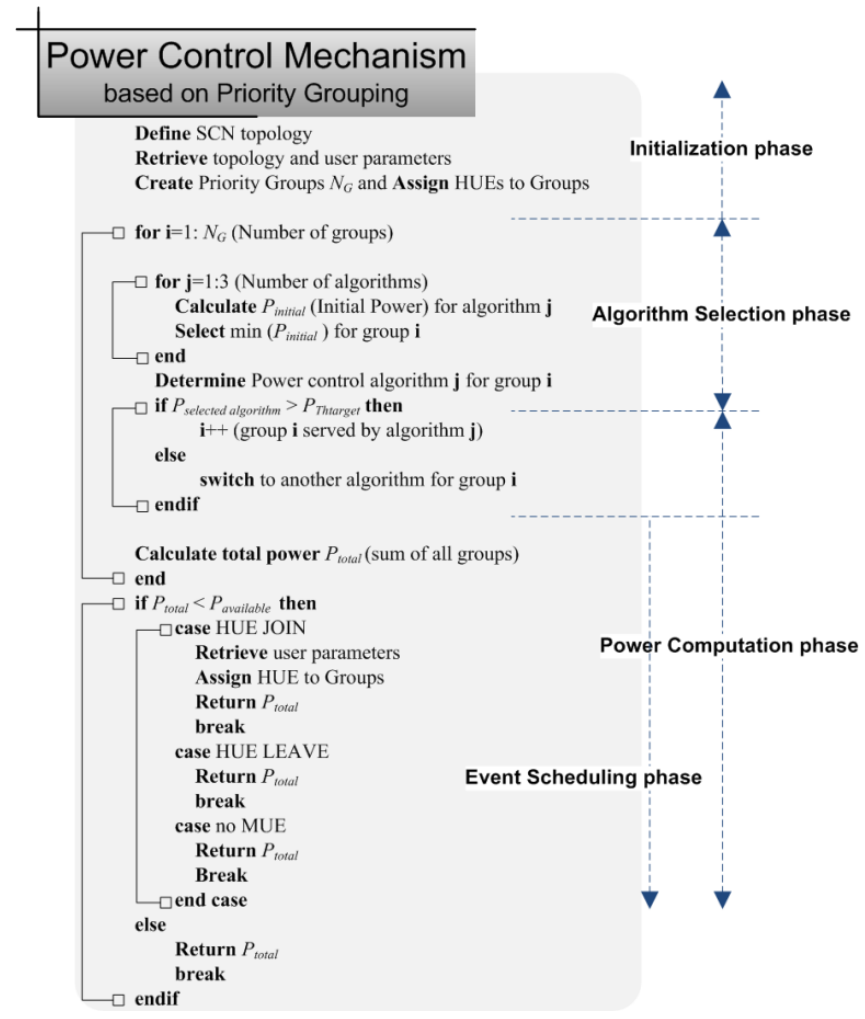

Figure 3. Pseudo code of the proposed mechanism

On stable state, in each group the algorithm that results to minimum total power of the HeNB is selected with respect to the performed priority grouping. In other words, in each group, the selected algorithm requires the minimum acceptable power in order to serve the total throughput of the HUEs that constitute each group.

Regarding the Event Scheduling phase, when any of the three different events occurred during a traffic scenario (HUE join/leave group, no MUE in the topology) a different procedure is triggered as depicted in Fig. 2.

The above description refers to a dynamic model as depicted in Fig. 2. Algorithm Selection phase is triggered whenever one of the three events of Event Scheduling phase is emerged. Furthermore, in a real world scenario, UEs are characterized by mobility. Consequently, the required power per group must be computed periodically every a predetermined frequency rate (fpower). This periodic computation inserts a further complexity in HeNB. However, it should be mentioned that the computation frequency (fpower) is beyond the scope of this paper and should be further studied.

\section{PERFORMANCE EVAlUATION}

\section{A. Simulation Parameters}

The simulation parameters that are necessary for the conduction of the experiment are presented in Table II. The SCN topology consists of multiple adjacent macro cells, multiple femto cells that are uniformly distributed in the network and multiple macro and home users (see Fig. 4). Macro users are uniformly distributed in the topology and they can move to any direction whereas home users are uniformly distributed to femto cells and they considered as fixed position users. The source code of the implemented mechanism is available in [15].

TABLE II. SIMULATION PARAMETERS

\begin{tabular}{lll}
\hline Parameter & Units & Value \\
\hline System bandwidth & $\mathrm{MHz}$ & 10 \\
Subcarriers & & 60 \\
Subcarriers' bandwidth & $\mathrm{KHz}$ & 375 \\
Carrier frequency & $\mathrm{MHz}$ & 2000 \\
Cell Radius & $\mathrm{m}$ & 250 \\
Correlation distance & $\mathrm{m}$ & 40 \\
Channel model & & $3 \mathrm{GPP}$ Typical Urban \\
Users' speed & $\mathrm{km} / \mathrm{h}$ & 3 \\
Path loss & $\mathrm{dB}$ & Cost 231 Hata Model \\
BS transmit power & $\mathrm{dBm}$ & 43 \\
HeNB max transmit power & $\mathrm{dBm}$ & 10 \\
HeNB min transmit power & $\mathrm{dBm}$ & -10 \\
Antenna Gain & $\mathrm{dBi}$ & 14 \\
Fixed Power for Algorithm 1 & $\mathrm{dBm}$ & -3 \\
HeNB operation mode & & Closed Subscriber Group \\
$N_{G}$ (priority groups) & & 3 \\
Group 1/Group2/Group3 $T h_{\text {target }}$ & $\mathrm{Mbps}$ & $1.2 / 0.2 / 0.05$ \\
\hline
\end{tabular}




\section{B. Experiment Results}

For the needs of results' presentation, we conducted two experiments. In the first experiment a 9 macrocell and 5 femto cell network with 90 MUEs, 50 initial HUEs and approximately additional 65 HUEs gradually distributed in time domain was considered (Fig. 4). In second experiment, a 9 macrocell and 5 femto cell network with 90 MUEs, 50 initial HUEs and approximately additional 500 HUEs exponentially distributed in time domain was considered (Fig. 7). In the first experiment, HUE population is increased with a constant rate throughout the whole simulation, while in the second experiment; HUE population remains constant in the first half of the simulation and increased rapidly in the second half of the simulation constituting an aggressive femto cell topology. In the remaining of this section, the proposed mechanism is also referred as algorithm 4 .

\section{1) First experiment}

Fig. 4 displays the overview of the entire SCN topology. In order to better present the results, we focus on femto cell number 11 of the topology depicted on Fig. 4. Fig. 5 presents the comparison of the mean throughput achieved by all the HUEs that reside in femto cell 11, as also the mean throughput achieved by each group of the algorithm 4 (proposed mechanism) in the same femto cell. Furthermore, Fig. 6 depicts the evolution of HeNB transmit power for each power control algorithm as well as the HUE population evolution per group in time domain. Additionally, Fig. 6 presents the algorithm that is selected each time for each group by our mechanism in order to perform efficient power allocation at HeNB.

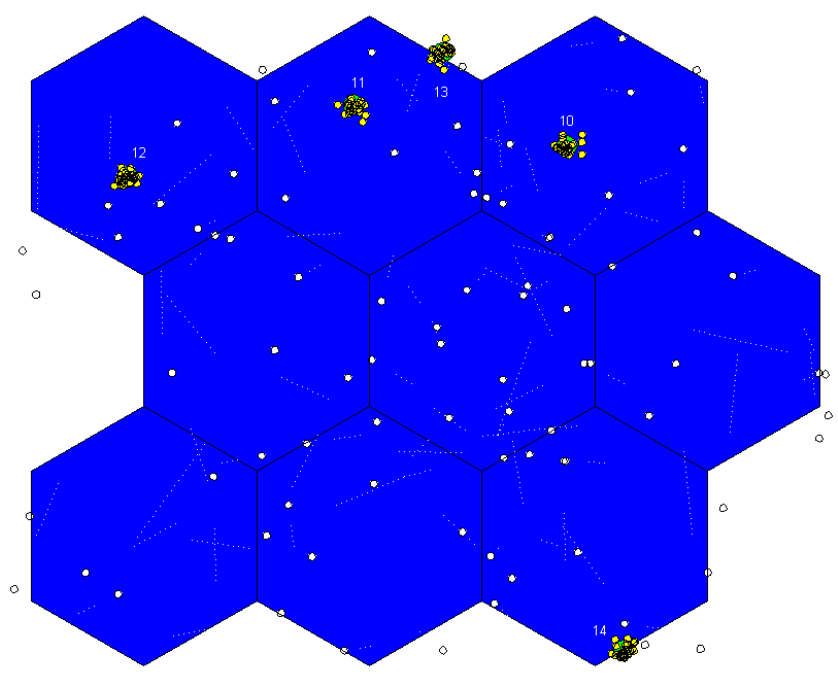

Figure 4. Topology for the first experiment

By combining the subplots of Fig. 6, we can assume that algorithm 4 (proposed mechanism) selects algorithm 3 for serving group 1 on timeframe $0-20 \mathrm{sec}$ then choose algorithm 2 for the rest of the experiment. This happens because in timeframe $0-20 \mathrm{sec}$, group 1 has small HUE population and most of the HUEs are located in femto cell edge, so high power is needed in order to achieve the target mean throughput of the group (1.2 Mbps see Table II). 20 sec later, although the HUE population of the group 1 starts rising, most of these HUEs are located in the center of femto cell 11. This means that less power is needed to support group 1 users and thus the proposed mechanism performs an algorithm switch from algorithm 3 to algorithm 2 (as shown in Fig. 6) so as to efficiently use the power resources.

Regarding group 3, Algorithm 4 choose algorithm 1 for serving the HUEs of the group on timeframe 0-5 sec then choose algorithm 2 on timeframe $5-10 \mathrm{sec}$ and then choose algorithm 1 again for the rest of the experiment. That happens because on timeframe $0-5 \mathrm{sec}$ there are no HUEs on group 3, on timeframe $5-10 \mathrm{sec}$ there are some HUEs mostly located on cell edge so the power has to be increased and finally, on the rest of the experiment the power of algorithm 1 is capable to serve the group 3 users with the target mean throughput (0.05 Mbps see Table II). Group 2 users (the target mean throughput $0.2 \mathrm{Mbps}$ see Table II) are efficiently served by algorithm 1 throughout the whole duration of the simulation.
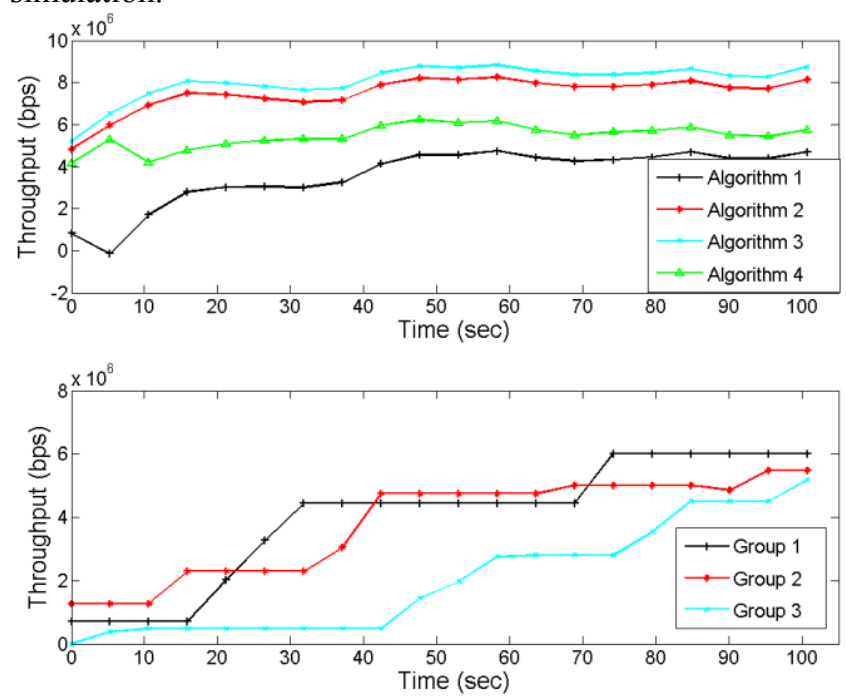

Figure 5. Achieved throughput for cell 11
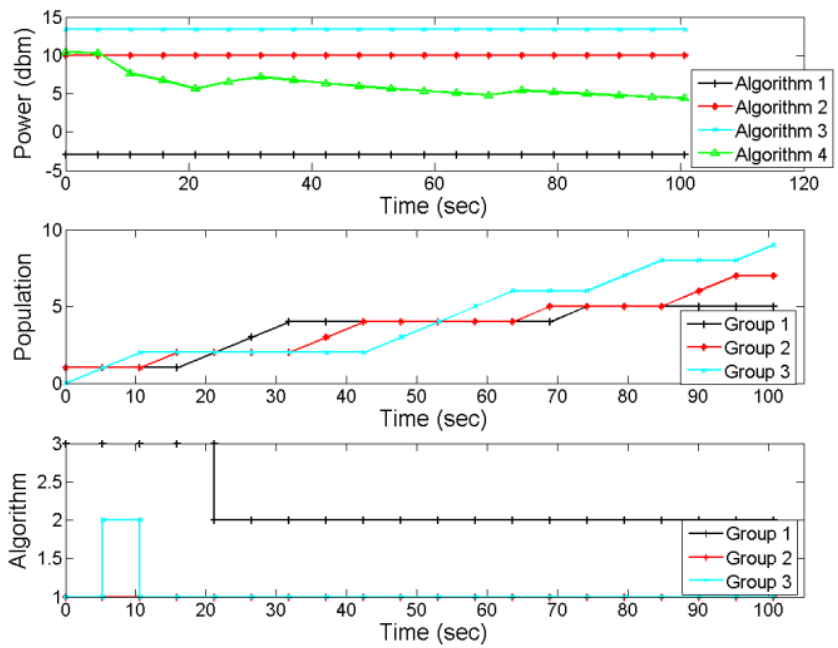

Figure 6. Power selection for cell 11 
Moreover, combining Fig. 5 and Fig. 6 we can see that even thought algorithm 1 achieves the lowest power from all the other algorithms, the mean throughput we achieves (approximately 4Mbps at the end of simulation) is lower than the target throughput needed to support the traffic load of group 1 (approximately 6Mbps at the end of simulation). This means that the traffic scenario cannot by served by the solely usage of algorithm 1 throughout the whole duration of the simulation. On the other hand, algorithms 2 and 3 are capable to serve the HUEs with respect to the target throughput but there is waste of power resources if we compare them with the proposed mechanism (algorithm 4). Algorithm 4 is capable to achieve the target throughput per group while on the other hand optimally handles the available power resources of HeNB. To conclude, the proposed mechanism results to mean power saving at HeNB over $38 \%$ compared to algorithm 2 and over $50 \%$ compared to algorithm 3 .

\section{2) Second experiment}

Fig. 7 displays the overview of the entire SCN topology for the second experiment. In this experiment we want to simulate an aggressive femto cell topology. For this purpose, HUE population remains constant in the first half of the simulation and increased rapidly in the second half. In order to better present the results, we focus on femto cell number 10 of the topology depicted on Fig. 7. Fig. 8 presents the comparison of the mean throughput achieved by all the HUEs that reside in femto cell 10 , as also the mean throughput achieved by each group of the algorithm 4 (proposed mechanism) in the same femto cell. Furthermore, Fig. 9 depicts the evolution of HeNB transmit power for each power control algorithm as well as the HUE population evolution per group in time domain. Additionally, Fig. 9 presents the algorithm that is selected each time for each group by our mechanism in order to perform efficient power allocation at HeNB.

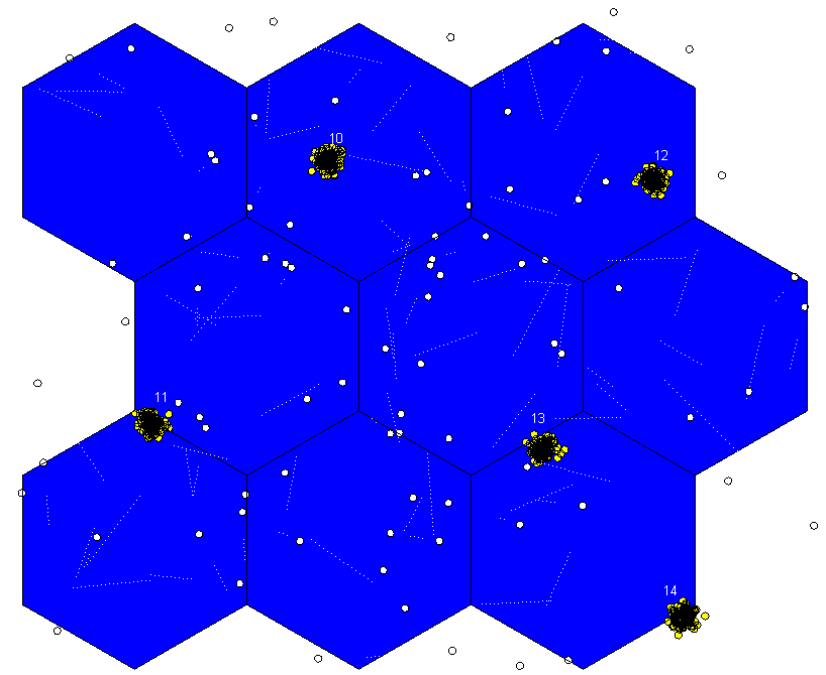

Figure 7. Topology for the second experiment

By combining the subplots of Fig. 9, we can see that the proposed mechanism (algorithm 4) choose algorithm 3 for serving group 1 at timeframe $0-6 \mathrm{sec}$ then choose algorithm
2 for the rest of the experiment. That happens because in timeframe 0-6 sec group 1 has small population and most of the HUEs are located in femto cell edge, so high power is needed in order to achieve the target mean throughput of the group (1.2 Mbps see Table II). $6 \mathrm{sec}$ after the beginning of the simulation, although HUE population of group 1 starts rising, most of these HUEs are located in the center of femto cell 10. This means that less power is needed to support group 1 users and thus the proposed mechanism performs an algorithm switch from algorithm 3 to algorithm 2 (as shown in Fig. 9) so as to efficiently use the power resources.
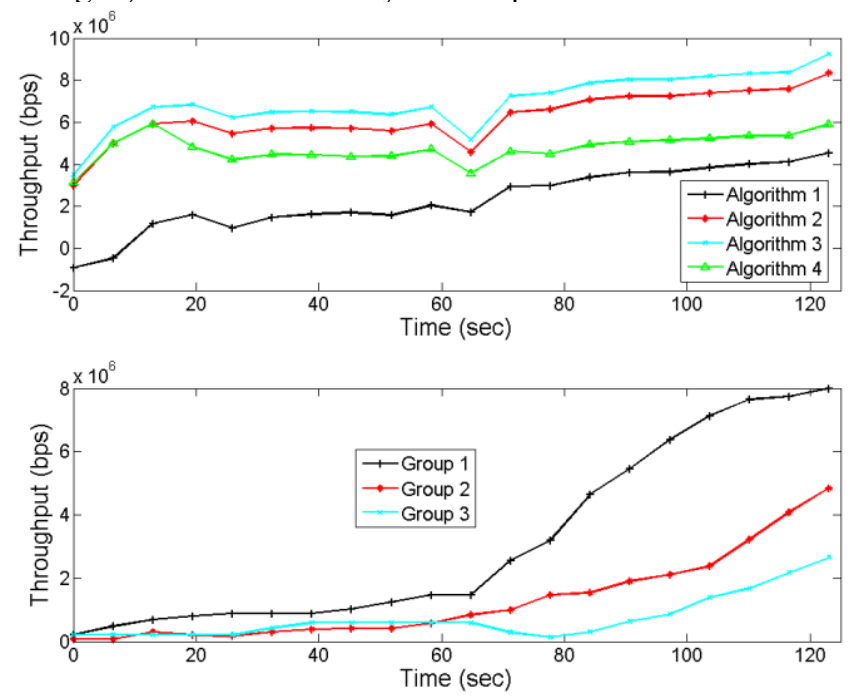

Figure 8. Achieved throughput for cell 10
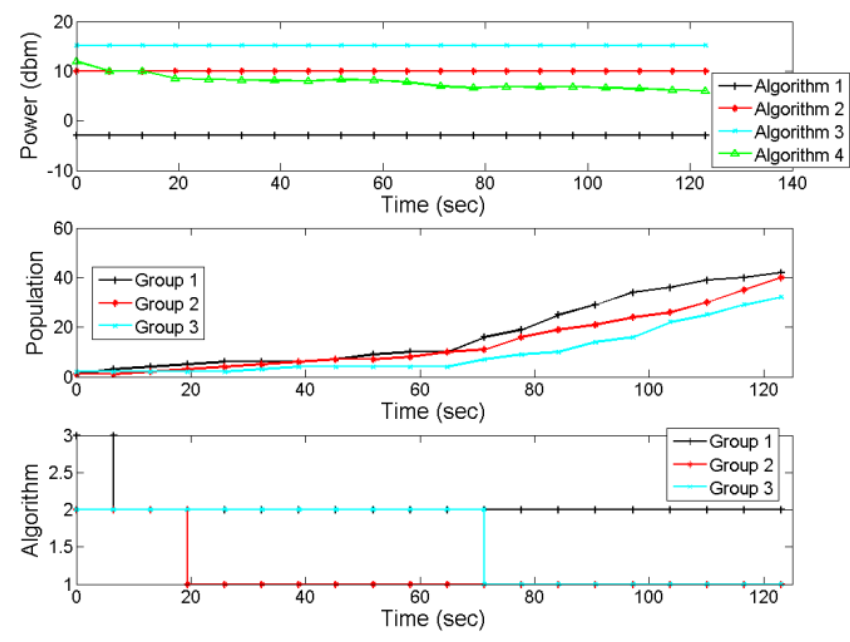

Figure 9. Power selection for cell 10

Regarding group 2, algorithm 4 firstly choose algorithm 2 for serving group 2 on $0-19 \mathrm{sec}$ and then switch to algorithm 1 for the rest of the experiment. That happens because on timeframe 0-19 sec, group 2 has small user population and most of these HUEs are located in cell edge, so high power are needed in order to achieve the target mean throughput (0.2 Mbps see Table II). After $19 \mathrm{sec}$, group 2 population start rising and most of these HUEs are located in cell center so less power is needed. Thus a switch to 
algorithm 1 is performed. On the rest of the experiment the power of algorithm 1 is capable to serve the group 2 users with the target mean throughput.

The proposed mechanism select algorithm 2 as starting algorithm for group 3 and keeps the same algorithm during the timeframe $0-70 \mathrm{sec}$. Then, it switches to algorithm 1 for the rest of the experiment. On timeframe 0-70 group 3 has small population and most of these HUEs are located in femto cell edge so high power is needed in order to achieve the target mean throughput (0.05 Mbps see Table II). At 70 sec, group 3 population start rising rapidly and most of these HUEs are located in cell center so less power is needed. Thus a switch to algorithm 1 is performed. On the rest of the experiment the power of algorithm 1 is capable to serve the group 3 users with the target mean throughput.

Moreover, combining Fig. 8 and Fig. 9 we conclude to the same observations as in the first experiment. Even thought algorithm 1 achieves the lowest power from all the other algorithms, he cannot achieve the required throughput in order to efficiently serve the traffic load of the scenario. Algorithm 4 is capable to achieve the target throughput per group while on the other hand optimally handles the available power resources of HeNB. The proposed mechanism results to mean power saving at HeNB over $21 \%$ compared to algorithm 2 and over $40 \%$ compared to algorithm 3.

Comparing the two experiments, we observe that the power saving in the second experiment is less than that of the first experiment. This happens because in the second experiment, we choose to simulate an aggressive femto topology in which HUE population is five times higher than that of the first experiment and also it increases rapidly compared to the first scenario.

\section{CONCLUSIONS AND FUTURE WORK}

In this paper, a power control mechanism for $\mathrm{SCNs}$ is presented. The mechanism efficiently controls the available power resources at HeNB and guarantees home user QoS. To achieve this, the methodology of Priority Grouping is introduced. With Priority Grouping, home users are assigned to one of the available groups with different priorities in terms of power requirements and requested traffic load. The proposed mechanism is evaluated through a user-friendly graphical tool designed to reproduce and calculate the optimal transmission parameters, via a graphical representation of the entire topology for a highly customizable network configuration. The results prove that the proposed mechanism can result to mean power saving at HeNB over $21 \%$ compared to existing $3 \mathrm{GPP}$ algorithms. The steps that follow this work could be at a first level the evaluation of the mechanism through additional simulation scenarios so as to estimate the algorithm switching points of the mechanism. This information could be used as feedback to the mechanism in order to improve its performance. At a second level, the complexity that the mechanism inserts in
HeNB due to its dynamic and periodic nature could be investigated.

\section{REFERENCES}

[1] J. Hoydis, M. Kobayashi, and M. Debbah, "Green small-cell networks," IEEE Vehicular Technology Magazine, vol. 6, no. 1, pp. $37-43$, march 2011.

[2] A. Valcarce, G. de la Roche, and Z. Jie, "OFDMA femtocells: A roadmap on interference avoidance," IEEE Communications Magazine, volume 6, Issue 9, pp. 41- 48, September 2009.

[3] M. Yavuz, F. Meshkati, S. Nands, A. Pokhariyal, N. Johnson, B. Raghothaman, and A. Richardson, "Interference management and performance analysis of UMTS/HSPA+ femtocells," IEEE Communications Magazine, vol. 47, issue 9, pp. 102 - 109, Sep. 2009.

[4] J. D. Hobby and H. Claussen, "Deployment Options for Femtocells and their Impact on Existing Macrocellular Networks," Bell Labs Technical Journal, vol. 13, no. 4, pp. 145-160, Feb. 2009.

[5] R.-T. Juang, P. Ting, H.-P. Lin, and D.-B. Lin, "Interference Management of Femtocell in Macro-Cellular Networks," in Proc. of the IEEE Wireless Telecommunications Symposium (WTS), Tampa, U.S.A., pp. 1-4, Apr. 2010.

[6] D. Lopez-Perez, A. Valcarce, G. de la Roche, and J. Zhang, "OFDMA Femtocells: A Roadmap on Interference Avoidance," IEEE Communications Magazine, vol. 47, no. 9, pp. 41-48, June 2009.

[7] Z. Bharucha, A. Saul, G. Auer, and H. Haas, "Dynamic Resource Partitioning for Downlink Femtoto- Macro-Cell Interference Avoidance," EURASIP Journal on Wireless Communications and Networking (special issue on Femtocell Networks), vol. 2010, no. Article ID 143413, May 2010.

[8] C. Bouras, G. Kavourgias, V. Kokkinos and A. Papazois, "Interference Management in LTE Femtocell Systems Using an Adaptive Frequency Reuse Scheme," Wireless Telecommunications Symposium (WTS 2012), London, UK, pp. 1 - 7, April 2012

[9] 3GPP TR 36.921 3rd Generation Partnership Project; Technical Specification Group Radio Access Network; Evolved Universal Terrestrial Radio Access (E-UTRA); FDD Home eNode B (HeNB) Radio Frequency (RF) requirements analysis (Release 11) V11.0.0 (2012-09)

[10] 3GPP TR 25.967 3rd Generation Partnership Project; Technical Specification Group Radio Access Network; Home Node B (HNB) Radio Frequency (RF) requirements (FDD) (Release 11) V11.0.0 (2012-09)

[11] S. E. Nai, T. Q. S. Quek, and M. Debbah, "Shadowing time-s cale admission and power control for small cell networks," in Proc. IEEE International Symposium on Wireless Personal Multimedia Communications, Taipei, Taiwan, pp. 628 - 632, Sep. 2012.

[12] V. Chandrasekhar, J. G. Andrews, T. Muharemovic, Z. Shen, and A. Gatherer, "Power control in two-tier femtocell networks," IEEE Trans. Wireless Commun., vol. 8, no. 8, pp. 4316-4328, Aug. 2009.

[13] F. Ahmed, A. Dowhuszko, and O. Tirkkonen, "Distributed algorithm for downlink resource allocation in multicarrier small cell networks," 2012 IEEE International Conference on Communications (ICC), pp. 6802 - 6808, June 2012.

[14] A. Barbieri, A. Damnjanovic, J. Tingfang, J. Montojo, W. Yongbin, D. Malladi, S. Osok, and G. Horn, "LTE Femtocells: System Design and Performance Analysis," IEEE Journal on Selected Areas in Communications, Vol. 30, Issue 3, pp. 586 - 594, April 2012

[15] A Power Control Mechanism Based on Priority Grouping, Source Code available at: http://150.140.8.26/ru6/sim_smallcell.zip 